\title{
A Semantic Web Approach for Built Heritage Representation
}

\author{
Stefano Cursi $^{1}$, Davide Simeone ${ }^{1(\sqrt{(凶)})}$, and Ilaria Toldo ${ }^{2}$ \\ ${ }^{1}$ Sapienza University of Rome, Rome, Italy \\ \{Stefano.cursi, davide.simeone\} @uniroma1.it \\ ${ }^{2}$ University of Southern California, Los Angeles, CA, USA \\ toldo@usc.edu
}

\begin{abstract}
In a built heritage process, meant as a structured system of activities aimed at the investigation, preservation, and management of architectural heritage, any task accomplished by the several actors involved in it is deeply influenced by the way the knowledge is represented and shared. In the current heritage practice, knowledge representation and management have shown several limitations due to the difficulty of dealing with large amount of extremely heterogeneous data. On this basis, this research aims at extending semantic web approaches and technologies to architectural heritage knowledge management in order to provide an integrated and multidisciplinary representation of the artifact and of the knowledge necessary to support any decision or any intervention and management activity. To this purpose, an ontology-based system, representing the knowledge related to the artifact and its contexts, has been developed through the formalization of domain-specific entities and relationships between them.
\end{abstract}

Keywords: Built heritage $\cdot$ Knowledge-based model $\cdot$ Ontology-based systems · Building information modeling $\cdot$ Semantic web technologies

\section{Introduction}

Architectural heritage is a substantial, dynamic repository of knowledge whose significance and authenticity must be preserved. Each heritage object, considered as a unique and irreplaceable source of aesthetic, historical, and cultural values, needs to be recorded and documented in order to prevent any loss or damage and ensure well-informed activities of maintenance, repair, and change of use.

The process of research and investigation of an heritage asset is a complex set of activities that produces a large amount of measured surveys, describing in detail the physical, dimensional, mechanical, and chemical configuration of an artifact at a given point of time (tangible knowledge), and other baseline data not collected through direct survey activities such as any historical, social, political, economic, and cultural issue related to its context (intangible knowledge), in a systematic way. Recording such a large stock of information is a prime responsibility of everybody involved in the conservation process and requires the participation of professionals from different fields of expertise and interests [1]. Moreover, records' accuracy and completeness are crucial 
for all the activities of investigation, intervention, and maintenance of historical buildings and any inconsistency or lack of knowledge can bring to irreparable damage to the artifact or, in the worst case, even to its loss.

The recent interest in the development of more suitable tools able to manage the knowledge related to architectural heritage is due to the actual difficulty that the actors involved in the conservation process find in accessing information related to the artifacts. In fact, information management in heritage processes is still mainly document-based and the representation of the artifacts is just the sum of documentation provided by different professionals and collected into different databases and, as a result, its comprehensive understanding is difficult to be gained. Therefore, built heritage representation usually suffers of:

- Difficulty in checking the information stored and finding errors and inconsistencies in the different data sets;

- Lack of integration, coherence, and coordination among different documentation sets generated by different activities during the heritage process;

- Poor information management with consequent lack or duplication of data;

- Actors' difficulty to share knowledge and to collaborate in the investigation and restoration phases.

Over the past few years, some research focuses on the use of Building Information Modeling (BIM) in order to achieve a more complete integration of tangible and intangible knowledge in built heritage representation. The chance to couple abstract properties, such as structural, thermal, chemical, historical. And temporal, with the geometrical representation of an object, typical of BIM systems, intended as object-based systems that employs an integrated data repository in order to generate, manage, and store building information and domain knowledge from conceptual design to demolition $[2,3]$, is the main feature of a potential instrument able to allow for different kinds of reasoning. In this kind of 3D knowledge-rich parametric models, the representation of building components is not only a coherent geometrical representation of the reality, but also solves the redundancy problems typical of $2 \mathrm{D}$ representations while parametric rules and constraints can facilitate the creation of a rich model. Furthermore, BIM, as a process data stream system able to provide a "complete, live, interactive, and accurate" description of the entire building and of the relationships between its elements in a dynamic virtual environment where any information can be constantly shared and transformed [4], is particularly suitable to represent and manage the changes that occur during time in architectural heritage. It can potentially allow for an effective exchange of information between different domains and platforms and for the creation of enriched models in which the level of detail of the objects from approximation moves to a more precise description.

Nevertheless, this representation system has its own limits. The parametric description of heritage objects in a BIM environment, at the current stage of its development, results problematic since BIM systems were originally conceived as an answer to the standardization of new construction elements. The uniqueness of heritage artifacts demands, instead, for the possibility to create in the model non-standardized objects with a customizable set of functional, semantic, and topological rules. 
In this context, the hereby presented research investigates the possible use of semantic web technologies in the perspective of providing a new modeling approach, able to comprehensively represent all the knowledge collected, used and shared during the activities of investigation, survey, intervention and conservation of an architectural heritage artifact.

\section{Previous Works on Built Heritage Representation}

\subsection{Virtual Reality GIS}

At present, Virtual Reality Geographic Information Systems (GIS) are the main tools used for storing and visualizing as geometrical information the geographical features, referring those data by means of map projections and 3D spatial coordinates.

One of the most widespread application of Virtual Reality GIS is the CityGML international standard, which is able to represent objects in relation to their geometrical, topological, semantic and appearance properties. This approach extends the capabilities of Virtual Reality modeling: if in the past 3D models have been used primarily for visualization purposes, with the addition of topology and semantic information, more complex analysis and 3D spatial queries can be carried out.

Although CityGML was designed for city modeling (e.g. for planning or disaster management), because of its capabilities and interoperable design, some studies have tested its effectiveness in built heritage modeling. For instance, D'Andrea [5] focused on future perspectives for archaeological documentation where CityGML was assessed for the management of spatial and geographic documents, while Delgado [6] adopted CityGML as an approach to semantic modeling of existing buildings in order to support intervention and monitoring of architectural heritage. Both research works show that an effective extension of Virtual Reality GIS framework to built heritage field cannot be achieved without the implementation in these models of additional, domain-specific semantics. In the typological classification of GIS, these application attempts on existing buildings matches a thematic and methodological distinction but not a technological innovation, at least for now.

In fact, despite the presence of some new VR GIS applications in this field, even with special interfaces and new algorithms, a specific modeling framework for built heritage representation has not yet been designed, except perhaps some specific intrasite applications. As a result, the standardization of VR GIS application to existing buildings is still far from having consistent results, hindered also by issues in terms of integration of VR GIS technologies with existing documentation and coding databases, both at regional and national level.

\subsection{Advantages and Limits of Building Information Modeling to Built Heritage}

Despite the growing interest of the AEC Industry in the use of Building Information Modeling to support life cycle, maintenance, and deconstruction management of new buildings, the research in BIM implementation for existing buildings is rather neglected. In literature, articles published in academic journals and applied publications that 
contribute to BIM implementation for existing buildings can be found starting from 2007, while the research on the use of BIM specifically for architectural heritage is a quite recent phenomenon and only a few publications in this field have been taken during the last few years. Nevertheless, the trend of BIM publication on this topic is significantly increasing.

Among all the papers related to the use of BIM as a support in the heritage conservation process, a consistent number of publications concentrates on the acquisition of the geometrical asset for existing structures through high-tech systems for field data acquisition, such as laser scanner and other photogrammetry techniques. One of the first applications of 3D geospatial information translation into an efficient 3D as-built BIM creation can be found in the concrete cracking analysis of the Solomon R. Guggenheim Museum in New York conducted by Robert Silman Associates in 2007 [7]. In order to keep track of concrete cracks and define their cause, the engineers worked with a digital survey company to create a full model of the building in a finite element modeling software. Measurements of the movements of the building have been collected for one year in order to calibrate the model and determine guidelines to settle the problem. This first experience pointed out the advantages of a rich 3D model integrated with specific information in the management of an existing building.

Starting from 2008, further studies have concentrated more on the development of enriched models adding intangible information to a component-based 3D BIM for heritage. Pauwels [8] proposed an approach similar to BIM, called Architectural Information Modeling (AIM), in which he theoretically describes a system obtained blending historical building information with a 3D model in a structure that can be implemented as soon as information is collected. At the Dublin Institute of Technology, Dore et al. [9] showed the advantages in using parametric and procedural modeling techniques typical of BIM systems for digitally recording architectural heritage. In their proposed model, named Heritage Building Information Modeling (HBIM) [10], a library of heritage objects has been designed following parametric rules and constraints deduced from historic manuscripts and architectural patterns book, which is able to automatically generate 3D models from the original survey data, allowing at the same time for manual refinements. Despite the interesting use of the Geometric Descriptive Language (GDL), the enrichment of the model with semantics, topology and appearance properties has not been made within BIM but through the transposition of the model in a 3D GIS environment where data related to built heritage artifacts have been used for further analysis.

These advances point out that the main advantage in the use of BIM for representing historical artifacts can be seen in the construction of a realistic 3D model able to store information related to each object such as morphology, type, construction data, etc.

Indeed, as for the AEC industry, BIM systems can ensure some benefits also in the built heritage field such as:

- Geometrical description of the object;

- A comprehensive and endowed description of each element by means of properties that can wide object's meanings;

- Semantic richness, able to provide different kinds of relationships which can be assessed for analysis and simulations; 
- Integration of data in a single repository ensuring their consistency, accuracy, and accessibility.

On the other hand, their application still have some limits:

- Many BIM's default families regard only modern and contemporary buildings. Therefore, they are not useful to describe existing architectures, which require the creation of ad hoc families;

- BIM software automatically applies to constructive elements some characteristics (e.g. the horizontality of the pavements or the regularity of the vertical surfaces) that do not regard heritage contexts;

- The asymmetric and geometrically irregular constructive elements of many historic buildings (as well as archaeological remains or fragments) are not compliant with the BIM features;

- Archaeological objects' modeling requires new libraries that contain specific families: they should be able to describe this domain and its peculiar characteristics.

Although these approaches are particularly suitable for achieving a realistic and substantial representation of the artifact, BIM and GIS systems, as data repositories, do not allow for any further kind of reasoning. Even if it is extremely useful to include all information in a single model all the information needed for a complete understanding of heritage assets, knowledge, meant as an awareness understanding of the artifact achieved through experience or education, cannot be exclusively associated to a BIM component.

At present, knowledge acquisition involves complex cognitive processes of perception, communication, and reasoning and is usually embed in designers' mind. Even if it is difficult to represent knowledge trough parameters, there are some application that can associate a higher level of semantics to each object, enhancing its understanding and helping professionals in the decision-making processes.

Over the past few years, in order to overcome these shortcomings and enhance the collaboration between different profiles operating in the heritage conservation fields, some research has been made on the development of integrated systems in which Building Information Modeling is combined with semantic web technologies, a technology already in use in the AEC industry for new constructions. As partially suggested by Pauwels [11], this kind of integrated model, in which information is digitalized, documented, archived, and enriched with a higher level of semantics, could also provide a valuable support in the process of investigation, interpretation, and conservation of architectural heritage increasing the level of knowledge of the artifact and its management.

\subsection{Current Knowledge Representation for Built Heritage Documentation}

The field of built heritage is certainly a context in which the use of Communication and Information Technologies, has found a strong distribution and use, as well as one of the disciplines in which there is more debate concerning which of them should be used and in which way. One of the main issues in their current applications is the representation of the many changes of the architectural artifact during both its life and the following process 
of conservation: the current method of building documentation in the Historic Structure Report (HSR) provides a significant amount of information but it is only a snapshot in time. It cannot respond to changes, renovations, and repairs. It does not serve as an up-todate reference for understanding the current state of a building. It is static and quite difficult to use since information related to different states of the artifact is spread in different archives and databases, usually not accessible, nor well-organized or structured.

In fact, current IT applications to heritage documentation process merely consist in the creation of large databases that have the task of gathering a wide range of information asynchronously provided by the different actors involved. This approach led to the proliferation of a wide variety of digital databases related to cultural heritage, mainly characterized by a great heterogeneity concerning the transmission formats used, the level of accessibility and the consistency of the information represented.

One of the first models for collecting information used in this context, perhaps the cause of most of the previously mentioned problems and limitations, is the recordoriented model based on "virtual catalog sheet" of objects, an approach directly derived from the traditional bibliographic one.

In this database, the information were represented mainly by textual descriptions of every single aspect of the object considered, thus preventing complex operations on data and resulting inappropriate for fully covering the complexity of historical objects and of their relationships.

This led to the creation of data models in which the information is fragmented in several basic entities, described by a list of properties associated with them, resulting in different computer applications based mostly on large relational databases. In this case, the main limitation continues to be the central role attributed to objects: the description of their features through a list of properties has led to a representation of the information often redundant and too simplified, highlighting the inability, through this data model, to represent semantic associations between objects and other parts of knowledge pertinent to other disciplines. It is therefore essential to use a technological infrastructure that allows to represent concepts relying on a knowledge representation and to perform high formal reasoning and inferences.

Ontologies are the primary tool used to overcome these problems, as they allow to represent objects and concepts not only through the description of their features but also by the description of the relationships that exist between them, paying attention to the meaning of the terms and the structure and nature of the different knowledge domain involved.

Several national and international organizations and commissions, including the ICCD (Central Institute for Cataloguing and Documentation) and the CIDOC (International Committee for Documentation of the Council of Museums) [12, 13] have focused their efforts towards the establishment of standards that indicated more precise and detailed criteria for structuring heritage-related knowledge. The CIDOC Conceptual Reference Model represents one of the main examples of this sort, as it provides a specific language for representing information on cultural heritage to mediate between different sources of information, such as the ones stored by museums, libraries and archives, also related to the concepts of space and time. While this model has been developed to manage cultural heritage documentation mainly for its cataloguing, other 
domain-specific ontologies have been progressively introduced to represent specific aspects of heritage conservation. The more emblematic is the Information System for Monument Damage Description (MONDIS) [14] that focuses on an ontological framework able to coordinate an automated reasoning behind the documentation of built heritage damages, their diagnosis and possible interventions.

Those examples show the potentialities of applying ontology-based models to heritage representation, documentation and analysis. Nevertheless, at present, these experiments are still too domain-specific and a more general knowledge-based model, able in particular to formalize in a homogenous way all the information related to an architectural heritage artifact, is still missing.

\section{A Knowledge-Based Model for Built Heritage Representation}

\subsection{Conceptual Framework and Research Methodology}

Investigating and operating on a built heritage artifact is a complex process where a large amount of heterogeneous data is collected, provided, used and shared by different specialists in many different ways.

The objective of this research was to develop a computational informative model for providing a homogeneous representation and integration of all the information collected and used during of the activities of investigation, intervention, use and management of heritage buildings. In the hereby presented model, a knowledge-based representation of different domains related to the heritage artifact and its multiple contexts, oriented to include and integrate any information necessary for its full comprehension, is proposed by means of ontologies. In the AEC field, several researchers have shown how ontologies can be effectively used to overcome the traditional difficulties of knowledge integration and sharing among the different specialists involved in an architectural design process [15-17].

The built heritage scope is not so different from the AEC ones. Several specialists with their own expertise, models, tools and jargon - are usually involved in the process of investigation and restoration of a Built heritage artifact, and each of them is both user and provider of a large amount of knowledge to share with the others actors involved. In addition, heritage artifacts usually require a wider knowledge base for their full comprehension, including crucial information such as those related to their historical context and their evolution along time. An ontology-based representation system, with its hierarchical and relational modeling features, is potentially able to represent in a consistent way all the knowledge derived from the different domains, and to integrate it in a single, coherent and up-to-date model. The main advantage of using ontologies is the ability of homogenously representing concepts, relationships, functions, rules and constraints in a unified semantic network. In fact, usually, concepts are indicated as specific nodes, while relationships are displayed as oriented interconnections/vectors between two concepts. Properties are variables' values stored in slots of each concept, while functions, rules and constraints are algorithms that can be executed to 'walk' through the semantic network in order to search, access, compute and verify the information stored. 
Indeed, ontologies have already been introduced in heritage conservation field as a way to represent and manage information and knowledge related to specific domains and disciplines (i.e. the previously mentioned CIDOC and MONDIS). Nevertheless, these models are very limited in terms of domain of application (historical documentation in the case of the CIDOC system, damage representation in the case of MONDIS) and can only partially being applied to Architectural Heritage representation. As a consequence, their being so domain-specific reduces one of the main potentialities of semantic web technologies, the possibility of enhance knowledge sharing and collaborations among different specialists. In this context, we illustrate a research aimed at using ontologies in order to develop a more general knowledge-based model able to integrate these domain-specific representations in a single, comprehensive relational database.

The presented research is mainly based on a constructive methodology: the general ontology-based modeling approach for architectural objects representation (and in particular on the one previously developed by the authors [18]) have been adapted to built heritage scope and then integrated with other domain-specific models. Where previous ontology-based models were not available, the conceptualizations implemented in the model have been developed by involving some specialists in the related field. This integration has been provided for peculiar fields such as the ones related to biological, chemical and microclimate analysis. After the construction of different semantic networks for the different knowledge domains considered, a process of mapping has been performed in order to depict the entities and the properties shared among different specialists and these entities behave as intersections among the different knowledge areas.

During the conceptual development of the modeling framework and the implementation of the platform by means of ontologies, the system has been progressively applied to the investigation process of the Castor and Pollux roman temple (located in Cori, a small town $50 \mathrm{~km}$ South of Rome). While the various survey and analysis were performed on the artifact, the platform was used to formalize and integrate the resulting information. As usual, the investigation process of the heritage artifact is asynchronous and spread along time; in this case, the proposed platform was able to represent also information provided by previous investigation activities and accessible through archival resources.

Despite the limitation of Building Information Modeling application for Built Heritage shown in the second chapter, a BIM platform has been used to provide a tridimensional representation of the physicality of the artifact and of its components, including also a representation of their properties. This connection between the database of the ontology-based system and the BIM database is possible because of their similar approach to the modeling of the architectural object. In fact, both of them rely on a component-based approach oriented to depiction of the single entities that compose the building (or, as in our case, the built heritage artifact) assigning to them a set of properties in accordance to the specifications of their class (defined as "families" in the BIM environment). Conceptually, this connection was provided by assigning the same label to both the representations of the entity in the two modeling environments (the BIM and the OWL database) and controlling the passage of information from one database to the other. As matter of fact, in this model the BIM platform was mainly used to display data and values stored in the ontology-based system. 
From the computational implementation perspective, the platform was developed by using the ontology-editor Protégé (4.3 version + WebProtégé for the final users' interface and VOWL for graphical visualization) while Autodesk Revit Architecture (2014 version) as BIM platform with the additional plugin DB-Link for the database exporting. For the connection between the two databases, an application prototype previously developed by the research group has been reused [13].

\subsection{The Knowledge-Based Model and Its Formalization}

Representation of existing and historical buildings information involves many and complex domains of interest in which is present both what UNECAP [19] defines as tangible and intangible knowledge. The previously presented analysis showed how the major limitations of most of the technologies used for this purpose are due to the difficulty of organizing a very large amount of data, usually extremely heterogeneous, without a structure that could underline the semantic links between the information. As mentioned above, because of the multitude of data with a very high semantic value and their particular aptitude to be interconnected, the Built Heritage field results to be an ideal candidate for the Semantic Web technologies application.

Ontologies allow users to exchange information in new ways and to create connections and understanding relationships that were previously hidden, revealing an added value otherwise invisible. Differently from their philosophical meaning, the ontologies used by the Semantic Web technologies only describe relationships between concepts that interest specific applications.

Defined by a set of primitives consisting of classes, properties and relationships (or rules), the ontologies, also due to their independence from lower level data models, are used for integrating heterogeneous databases, enabling interoperability among disparate systems and specifying interfaces to independent, knowledge-based services [20]. Moreover, the definitions of the representational primitives include information about their meaning and constraints and their logically consistent application.

As mentioned above, any ontological entity involved is fully described through three main features representing a synthesis of the concepts necessary for its formal characterization. By defining a class and thus assigning to it different meanings we can give a description of the entity in relation to the different knowledge domains considered; properties, instead, are necessary to represent all the descriptive aspects related to the concerned element such as geometrical, physical and behavioral features; rules, finally, can be divided into relationship rules and reasoning rules.

The relationship rules can link lower level entities and higher complexity ones as results of multiple entities' assembly, ('Part-Of', 'Whole-Of') or define hierarchical relationships ruling entity generality stratifications in terms of Father/Son, Prototype/ Instance ('Is-A', 'Instance-Of').

This kind of knowledge formalization is capable of characterizing the entities linked through the relations that occur among them, and which belong to the family of logical descriptions. In this way, we are able to manipulate also the type of an entity's structure, allowing not only to change the inheritance of an entity but also to mix entity assemblies. 
Reasoning rules can be made of: algorithms and formal codes for analyzing, checking and evaluating concepts associated to specific entities with inferential procedures of 'IfThen' type; cardinality constraints defined for each class; consistency rules formalized to check values, parameters, attributes, instances, relationships and properties referring to the specific meanings associated to each entity in the specific context in which it is used; empirical rules, best practice codes and concepts that represent part of the reasoning process of any actor on his/her own specific disciplinary domain during the investigation, interpretation an intervention process.

By means of this reasoning layer, able to match rules among the considered entities belonging to the same ontology, the system allows operators to use in a coherent manner different levels of abstraction and to exploit a real interoperability of concepts.

\subsection{The Knowledge Domains}

In order to provide a comprehensive and accurate description of a Built Heritage artifact and of its multiple contexts, we decided to depict and formalize all the conceptual entities considered necessary for its representation, and to classify them within four knowledge macro-domains: the Artifact, the Historical Context, the Heritage Process and the Actors, mutually interconnected by strongly structured relationships.

The model is based on the assumption that the representation of the involved knowledge domains is the result of a complex, dynamic and interactive system of many modeling entities - some related to physical objects, others to abstract concepts - each characterized by a class definition, a set of properties and a set of relationships with other entities.

The Artifact Domain. With reference to the architectural object, the heritage artifact has been investigated and modeled by relying on the definition of two macro-systems: the 'Spatial Class' and the 'Technological Class'. The first includes the spaces delimited by physical elements and their aggregations, while the second embeds all the physical elements (components) and their aggregations, which make up the constructive aspect of the artifact [21].

All the Classes belonging to the artifact knowledge structures have been defined by means of two iterative processes:

- an inductive approach in which entities have been first defined in terms of meaning, performances and requirements, and then instantiated by assigning all the required values and establishing connections, relationships and rules between them;

- a deductive approach in which every actor associated meanings, properties and rules to each entity starting from their geometrical definition.

All the identified entities has been agreed with a group of specialists in the restoration area in order to use definitions, methods and representative classification methodologies already consolidated in this field as those provided by the ICCD cataloging scheme. This correspondence aims at ensuring the consistency of data with the standards defined at a national level, thus guaranteeing the uniformity of the information, considered indispensable for correct usage and sharing among the specialist involved in the conservation process. 
Furthermore, the freedom ensured by this structure enables to compose an entity of a class also with entities of other classes belonging from heterogeneous domains (for example an entity belonging to the class of the spaces with one belonging to the one of the components). In our case, we have implemented the structure of spaces which, together with the system of components, contributes to completely define the building; so that the two structures (usually separated) can be interfaced directly through an inversion of the inheritance relationship (Is-A), allowing an assembly of mixed entities by means of the composition relationships (Whole-Of/Part-Of) (Fig. 1).

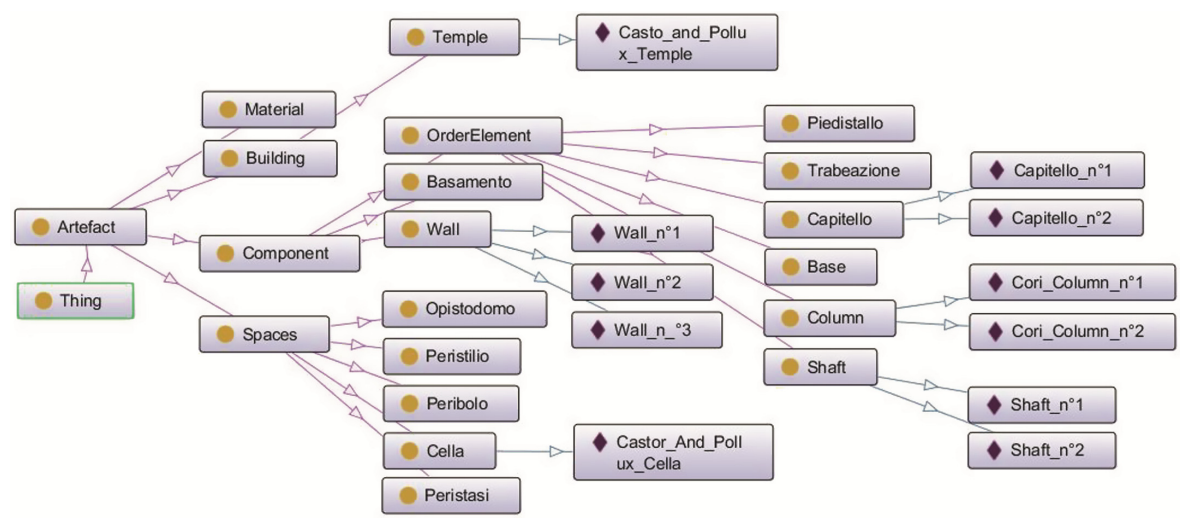

Fig. 1. Artifact knowledge domain modeled by means of ontologies - Spaces and Components classes (yellow bullets) are represented through the 'Is-A' relationship, while Individuals (purple diamonds) are linked to the class they belong through the 'Instance-Of' relationship (Color figure online).

In the presented case study, the definition of the artifact knowledge structure started with the formalization of the classic elements that are present in a Roman Temple, both in terms of spaces (e.g. Cella, Pronaos, Peribolos, etc.) and in terms of components (e.g. Column, Capital, Architrave, etc.) with the related construction materials. Subsequently, starting from the defined classes, the temple specific spatial and constructive entities deduced from the geometric survey - have been instantiated in the ontology editor and related each other through the assembly properties.

Since is not always possible to univocally identify the different components of ancient buildings, as often happens at the beginning of an investigation process, it has been necessary to develop some classes in order to represent unknown objects in terms of their geometric and material features, waiting for a later interpretation that will associate them to the proper class.

By assigning an entity to a class, it inherits all the properties of its representation template helping the specialist to understand the already available information and which data are still missing; thus, operators can see which entities are still unidentified and, on the bases of their knowledge and experience, provide suggestions for their interpretation (Fig. 2). 


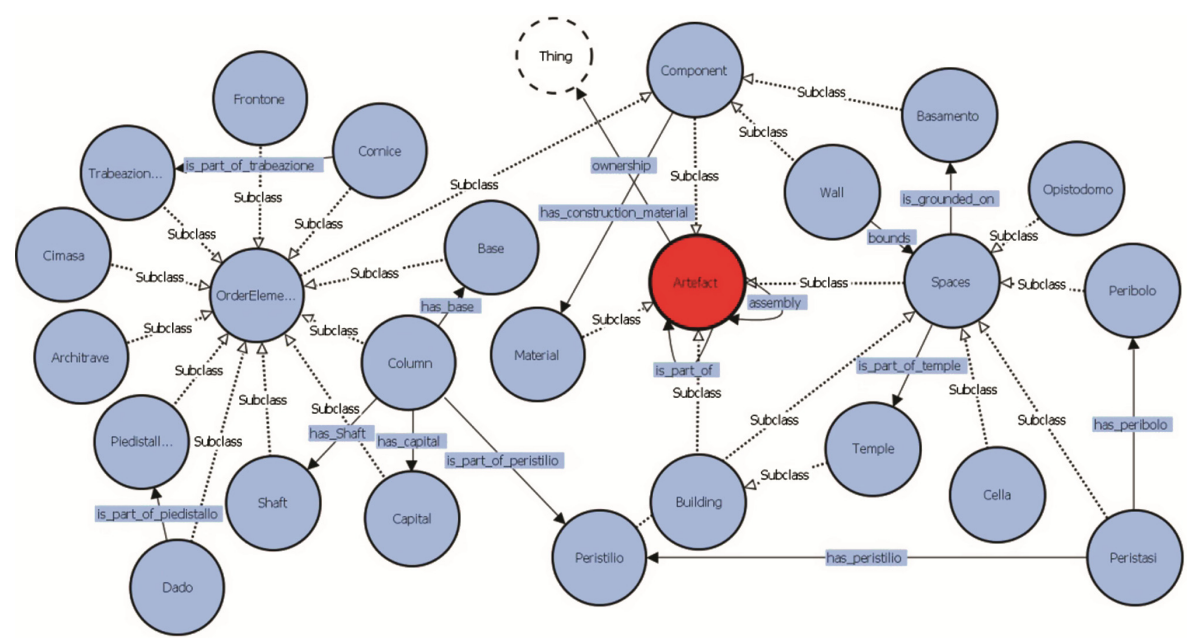

Fig. 2. Space and Component system of a classic Roman temple modeled through the assembly relationships (Part-Of/Whole-Of).

The Historical Context Domain. To fully understand a built heritage artifact, a representation of the current state of the object is not sufficient. Instead, it is necessary to include other intangible aspects such as its evolution during time, its historical, social and technological contexts, its intended functions and uses along time, the origin of the construction materials and other information usually collected by studying the archival sources.

The representation of physical evolution and functional architectural artifact, or rather the modifications that this has undergone over time, have been represented within the model through a set of classes defined Historical Context Entities.

The structure of this macro-domain has been designed by adapting the CIDOC-CRM international standard, nowadays largely accepted as a reference system for structuring the documentation of cultural heritage and by establishing interconnections and interoperability between different data structures.

The driving principle is the explicit modeling of Event entities (e.g. Beginning/ Ending of Existence, Transformation, Modification, etc.) to be linked with the concepts of Space-Time, Material/Immaterial Object in order to allow the formalization of metadata and the connection of facts into a coherent representations of the history.

The normal human way to analyze the past is to split up the evolution of matters into discrete events in space and time. Thus the documented past can be formulated as series of events involving Persistent Items like Physical Things and Persons, while Conceptual Objects are items that can be created but can reside on more than one physical carrier at the same time (e.g. a book, a computer disk, a painting, etc.), including human thought.

Only Temporal Entities - such as Events - can be linked to time while other objects (Conceptual and Physical Actors/People and Places) cannot be directly linked to time.

Physical Things are destroyed when they cease to be functional and therefore destruction is not necessarily linked to physically disappearing. A thing could be 
physically destroyed and transformed into something else preserving parts of it. That new thing then becomes part of our domain of interest (Fig. 3).

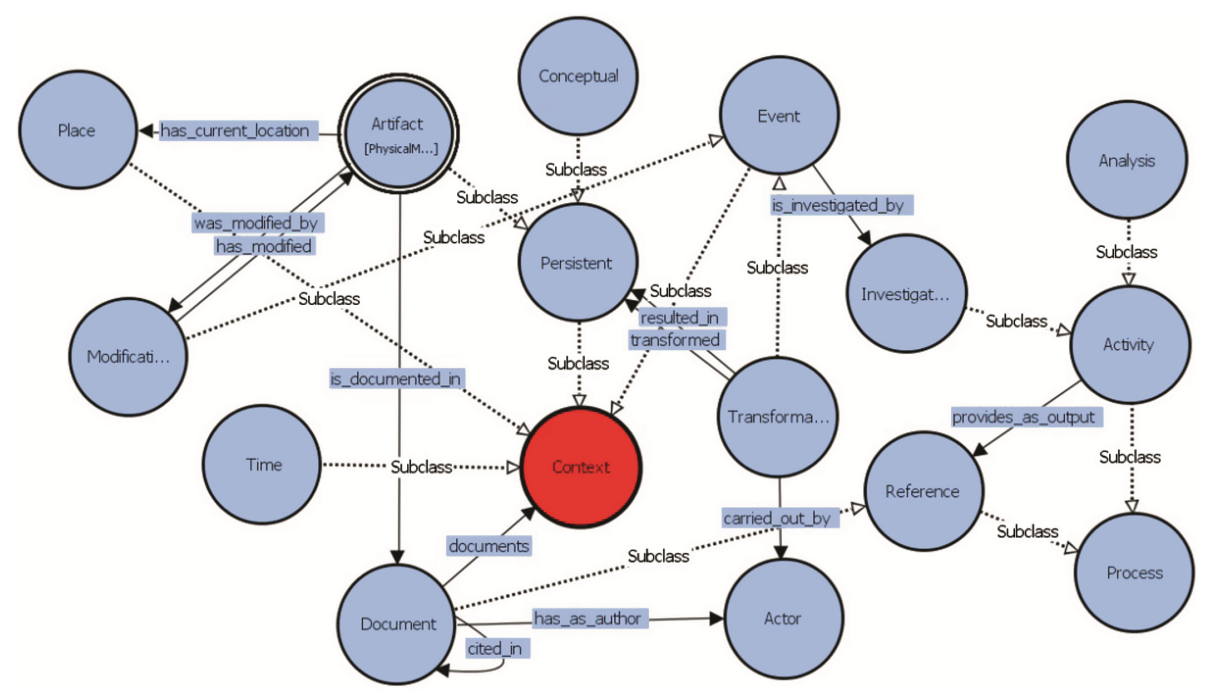

Fig. 3. Model of the historical building life-cycle in its physical and functional evolution together with the representation of the related archival sources.

Starting from the event of its construction (formalized by means of the Beginning of Existence) the case study temple has been modeled as a Physical Man-Made Thing (subclass of Persistent Item and equivalent to the Artifact Macro Domain). Then, through the Transformation and Modification classes (sub-class of Event) all its different configurations over the centuries have been represented, including for instance its transformation into a house during the middle ages. The changes of physical configuration have also been represented in the BIM environment, so that the different components of the different configurations can be filtered in accordance to the selected time span.

In fact, it was necessary to represent complex aspects such as morphology mutations, use changes and even destructions or relocation of building elements; for instance some columns of the case study were moved from their original position during time and reused in the medieval age as part of new walls. For this purpose, a specific set of dataproperties has been implemented in order to represent if the element is in place or not, the age, the period of realization, and the different phases of the heritage object life it was in.

Another relevant part of the Historical Context Domain is the representation of archival sources. Although in the CIDOC template there is a class defined as Document (subclass of Information Object) and a specific property 'documents/is documented in' that allow to connect an entity with a document, this representative structure does not allow to model the authors of the document, nor other elements such as citations and scholars of the source. 
In fact, in order to model the study of the artifact it is necessary to represent not only the sources that document the object, but also subsequent studies in which these documents are mentioned and/or used as a reference. This allows to model different studies and assumptions of various interpretations, representing the knowledge that will be the basis of an object evaluation/definition.

For this purpose, in the proposed model, the Document entity, together with the properties 'refers to', 'carried out by' and 'cited in', has been conceived as a bridge that allows the connection of the knowledge domain concerning the history of the artifact with the domain of its process of investigation, study, analysis and interpretation.

In order to facilitate the connection of such documents with the proper authors, it has been introduced the 'has as author' property (Fig. 4).

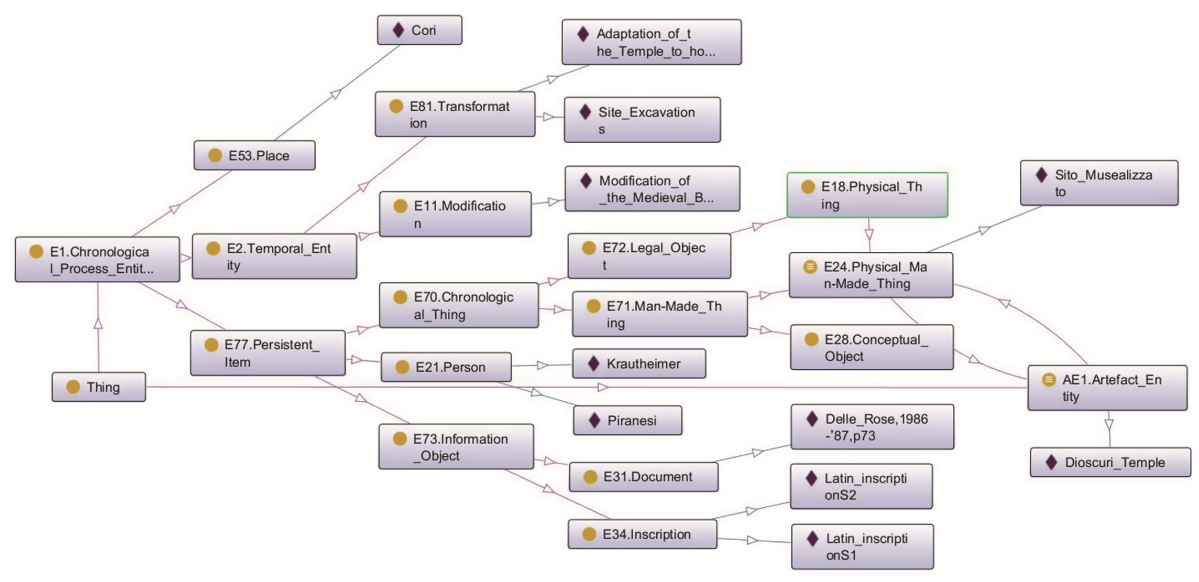

Fig. 4. Historical Context domain modeled by means of ontologies - Events and Persistent Item classes (yellow bullets) are represented through the 'Is-A' relationship, while Individuals (purple diamonds) are linked to the class they belong through the 'Instance-Of' relationship (Color figure online).

The Heritage Process Domain. In this model, the formalization of the knowledge concerning the macro-domain of heritage conservation and restoration processes comprises all the activities of investigation, analysis, and interpretation related to the artifact.

While, on one hand, the application of CIDOC system has allowed for testing its potential in modeling the knowledge associated to the artifact's life cycle, on the other hand, it has shown its limits in the representation of study, analysis, investigation, survey, and intervention activities, not just for the ongoing ones, but also in recording the previous ones. Concerning the historical documentation, the entities are points of conjunction with the classes related to the heritage process.

In analogy to the Historical Context, four main components have been identified and subsequently formalized in order to represent the Heritage Process domain: Heritage Process Activities, Resources, Actors, and Reference Information Objects. 
With the aim of following the logical steps carried out by the operators during an historical building investigation, we have chosen to formalize the activities related to the investigation, analysis, and interpretation as subclasses of the process activity domain.

Resources entities were introduced to represent tools, methodologies and samples used by operators during the activities described, while the reference information objects represented information and concepts used as input and provided as output during the various activities.

Through specific Interpretation Activity classes such as the Attribute Assignment and Appellation Assignment, it is possible to connect the activities of investigation and analysis to the possible interpretations and deductions, which edit and/or increase the knowledge concerning an entity of the artifact (Fig. 5).

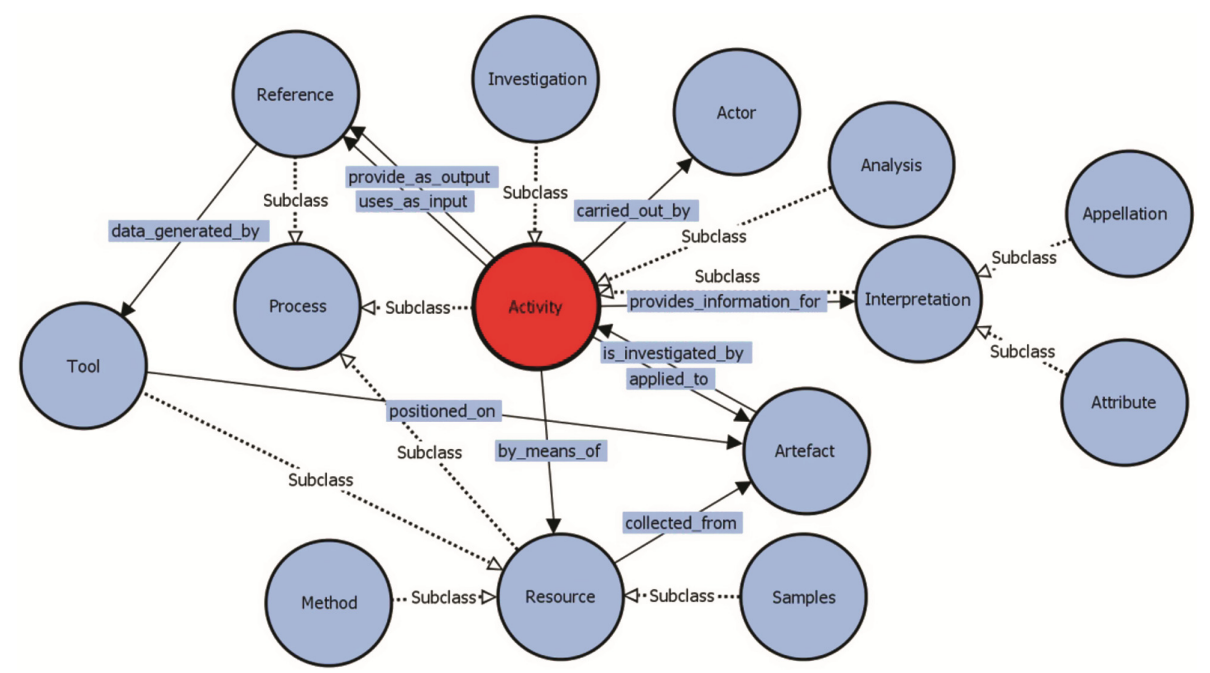

Fig. 5. Modeling framework of the investigation, analysis and interpretation activities related to the Heritage Process knowledge domain.

Particular relevance has been given to the Attribute and Appellation classes, whose purpose is to assign a definition/interpretation (respectively in terms of meanings and data) to an artifact entity. In this way, it is possible through an appropriate rule of reasoning to highlight the presence of multiple definitions assigned to an object, thus signaling problems or inconsistencies in its interpretation. Those entities must be associated with a suitable data property that could represent a range in the reliability of the attribution/definition.

Furthermore, in order to connect the different components of the artifact and the related knowledge, it has been necessary to model a set of properties related to the Heritage Process domain with a sufficiently generic range of application (Fig. 6). 


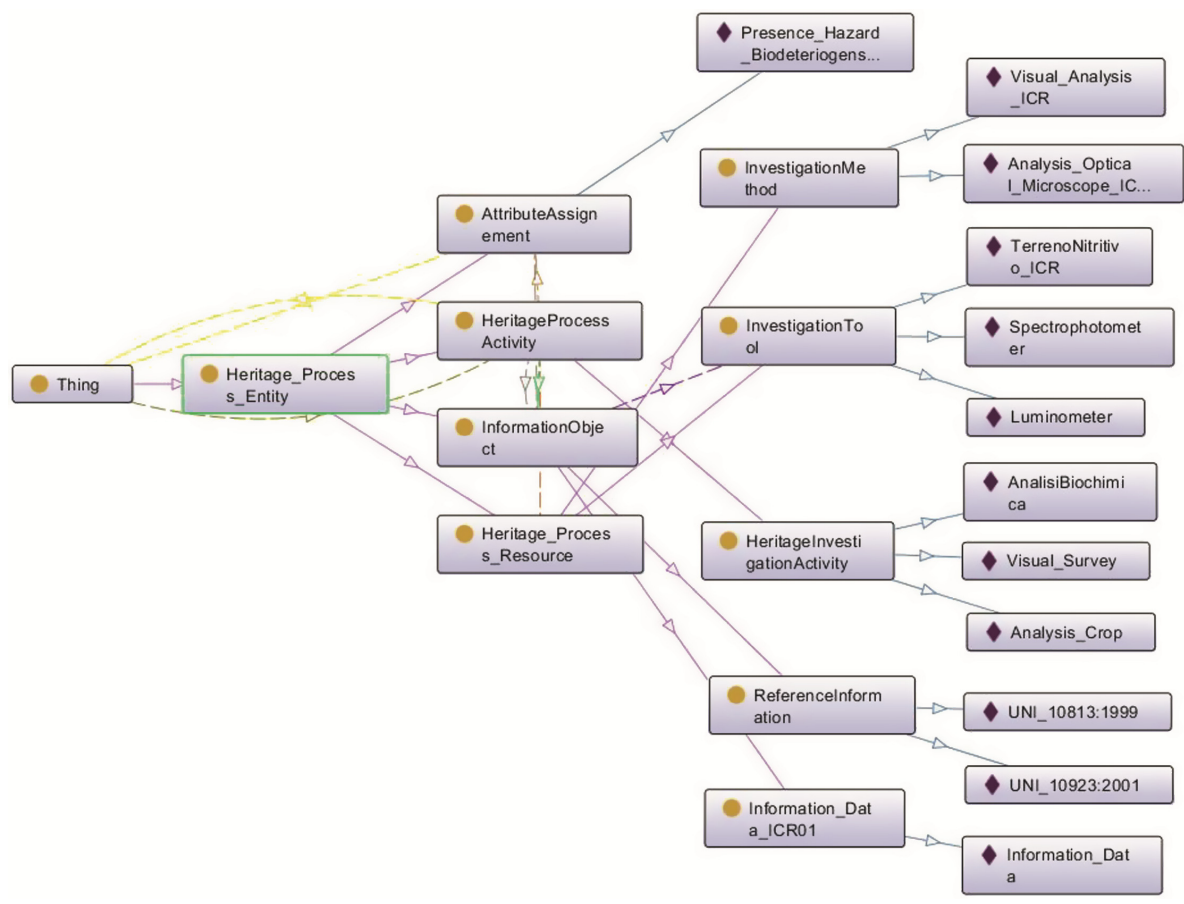

Fig. 6. Heritage Process domain modeled by means of ontologies - Activities, Resources, Information Objects and Attribute Assignment classes (yellow bullets) are represented through the 'Is-A' relationship, while Individuals (purple diamonds) are linked to the class they belong through the 'Instance-Of' relationship (Color figure online).

The Actors Domain. This domain includes all the actors that interact with the building during the conservation process and its possible future users.

We chose to model the operators in a separate domain although, as highlighted in the previous paragraphs, they are among the entities involved in the representation of both the Heritage Process activities and the Events of the Historical Context knowledge domain.

Formalizing the actors in a dedicated domain is mainly due to the necessity of knowledge management. In fact, in order to improve collaboration among the specialists involved in the process activities, the actors are required to interact with the entities of the other domains and control their specific features in relation to their needs.

To do so, the proposed template for knowledge modeling represents each entity primitive by different actors' perspective: by using this model, architects as well as conservationists can represent entities in terms of their discipline-specific definition (since the same entity can have different meanings according to different actors), properties and rules, pointing out special requirements and intents. At the specialist level, the right formalization of each actor's knowledge helps him/her in checking the coherency, congruence, and consistency of the ongoing investigation, interpretation, and intervention activities. 
By means of this formalized knowledge structure, collaboration, intended as "problem sharing", is enhanced as common concepts and entities are shared and agreed by all the actors involved, helping avoid misunderstandings, misinterpretations and lacks of data.

\section{Conclusions and Future Developments}

In this paper, we presented the results of a research conducted through a constructive methodology and aimed at conceiving an ontology-based model for Built Heritage knowledge representation. The presented model was developed by adapting to the Built Heritage scope the conceptual framework of an ontology-based modeling approach already well-known in AEC field. In fact, while ontologies have shown their great potentials in supporting knowledge representation and sharing in new building design processes, no attempts were made for their application to the built heritage field. By integrating this knowledge structure with the semantic networks related to some of the different disciplines involved in a heritage investigation process and by mapping the entities (physical or abstract) shared among them, we were able to generate a semantically rich representation of the artifact and of the knowledge necessary for its full comprehension.

This is a first, innovative step in built heritage information management field; it means moving from a documentation-based informative system, where information about the artifact is stored in incremental, not structured archives (or databases) to a model-based system, where every piece of information is a part of a coherent, interconnected and up-to-date representation.

The proposed model provides a solution to several problems of current informative systems, such as inconsistency and duplication of data, or incoherence between different representations of the same aspects of the built heritage artifact, are solved.

On the other side, the introduction of this modeling approach opens for the necessity of addressing new issues in terms of knowledge managing protocols and filters. Among the others, we can cite the relevant topic of "property" of the knowledge shared through this system, or the one related to representation of reliability of information, that in built heritage processes are often results of interpretation or interpretative analysis. Among the others, our current work is focusing on improving semantic filtering between different domains and disciplines in the heritage practice, and on investigating how Building Information Modeling approach and the Knowledge-based one can be integrated more effectively.

Acknowledgements. This research was partially supported through a Research Project of National Interest ("Built Heritage Information Modelling/Management - BHIMM", 2012) funded by the Italian Ministry of Education University and Research.

The authors are grateful to Gianfranco Carrara from Sapienza University of Rome and Karen Kensek from University of Southern California for supporting this research with useful comments, suggestions and feedbacks. 


\section{References}

1. Letellier, R., Schmid, W., Le Blanc, F.: Recording, Documentation, and Information Management for The conservation of Heritage Places Guiding Principles. The Getty Conservation Institute, Los Angeles (2007)

2. Lee, G., Sacks, R., Eastman, C.M.: Specifying parametric building object behavior (BOB) for a building information modeling system. Autom. Constr. 15, 758-776 (2006)

3. Eastman, C.M., Teicholz, P., Sacks, R., Liston, K.: BIM Handbook: A Guide to Building Information Modeling for Owners, Managers, Designers, Engineers and Contractors. Wiley, New Jersey (2011)

4. Kensek, K.M.: Building information modeling. In: Smith, E.R. (ed.) Pocket Architecture: Technical Design Series. Routledge, Taylor \& Francis Group, London (2014)

5. D'Andrea, A.: Sharing 3D Archaeological Data: Tools and Semantic Approaches. In: 14th International Conference on Virtual Systems and Multimedia, Limassol, Cyprus, pp. 149_ 156 (2008)

6. Delgado, F., Martínez, R., Hurtado, A., Finat, J.: Extending functionalities of Management Systems to CityGML. In: eWork and eBusiness in architecture, engineering and construction, pp. 409-415, CRC Press (2010)

7. Ayón, A.: Historic fabric vs. design intent: authenticity and preservation of modern architecture at Frank Lloyd Wright's Guggenheim Museum. J Archit. Conserv. 15, 41-58 (2009)

8. Pauwels, P., Verstaeten, R., De Meyer, R., Van Campenhout, J.: Architectural information modelling for virtual heritage application. In: Digital Heritage, 14th International Conference on Virtual Systems and Multimedia, Lymassol, Cyprus, pp. 18-23 (2008)

9. Dore, C., Murphy, M.: Integration of historic building information modeling (HBIM) and 3D GIS for recording and managing cultural heritage sites. In: Virtual Systems in the Information Society, VSMM 2012, 18th International Conference on Virtual Systems and Multimedia, Milan, Italy, pp. 369-376 (2012)

10. Murphy, M., McGovern, E., Pavia, S.: Historic building information modelling (HBIM). Struct. Surv. 27, 311-327 (2009)

11. Pauwels, P., Bod, R., Di Mascio, D., De Meyer, R.: Integrating building information modelling and semantic web technologies for the management of built heritage information. In: 1st International Congress on Digital Heritage, Marseille, France, pp. 481-488 (2013)

12. The CIDOC conceptual reference model, references http://www.cidoc-crm.org/ references.html

13. Doerr, M.: The CIDOC conceptual reference module: an ontological approach to semantic interoperability of metadata. AI Mag. 24(3), 75-92 (2003)

14. Cacciotti, R., Blasko, M., Valach, J.: A diagnostic ontological model for damages to historical constructions. J. Cult. Heritage 16(1), 40-48 (2015)

15. Beetz, J., van Leeuwen, J.P., de Vries, B.: An ontology web language notation of the industry foundation classes. In: the 22nd CIB W78 Conference on Information Technology in Construction, CIB-W78, Dresden, Germany, pp. 193-198 (2005)

16. Carrara, G., Fioravanti, A., Loffreda, G., Trento, A.: Conoscere Collaborare Progettare, Teoria tecniche e applicazioni per la collaborazione in architettura. Gangemi Editore, Roma (2014)

17. Yongwook, J.: Mediating semantics for multidisciplinary collaborative design. $\mathrm{PhD}$ dissertation, Berkeley University of California (2008) 
18. Simeone, D., Cursi, S., Toldo, I., Carrara, G.: BIM and Knowledge Management for Archaeological Heritage. In: ACADIA 14: Design Agency - Proceedings of the 34th Annual Conference of the Association for Computer Aided Design in Architecture, Los Angeles, pp. 681-390 (2014)

19. UNECAP: Cultural Tourism Sites Management - A Training Manual for Trainers in the Greater Mekong Subregion. United Nations, Economic and Social Commission for Asia and the Pacific (2008)

20. Gruber, T.: Ontology, Encyclopedia of Database Systems. In: Liu, L., Tamer, M.O. (eds.) pp. 1963-1965 (2008)

21. Carrara, G., Fioravanti, A., Novembri, G.: Knowledge-based System to Support Architectural Design. In: Penttila, H. (ed.) Architectural Information Management, Proceedings of eCAADe 01 Conference, Helsinki, pp. 80-85 (2001) 